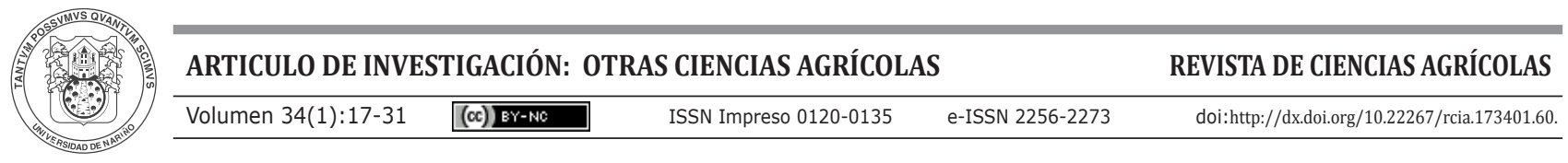

\title{
Mecanismo de acción de cinco microorganismos promotores de crecimiento vegetal
}

\author{
Action mechanism of five microorganism promoters of plan growth
}

\author{
Harold González F. ${ }^{1}$; Natalia Fuentes M. ${ }^{2}$
}

1 Docente. Microbiólogo. Instituto Nacional de Formación Técnica Profesional - INFOTEP. San Juan del Cesar. La Guajira, Colombia, hgonzalezf@infotep.edu.co

2 M.Sc. Ciencias Ambientales, Grupo de Investigación Ciencia y Ambiente, Universidad de La Guajira, Riohacha, La Guajira, Colombia..

Citar: GONZALEZ, H.; FUENTES, N. 2017. Mecanismo de acción de cinco microorganismos promotores de crecimiento vegetal. Rev. Cienc. Agr. 34(1): 17-31. doi: http://dx.doi.org/10.22267/rcia.173401.60.

Fecha de recepción: Julio 16 de $2015 . \quad$ Fecha de aceptación: Febrero 04 de 2016.

\section{RESUMEN}

El uso indiscriminado de agroquímicos para aumentar la productividad de los cultivos, es una práctica no amigable con el ambiente. Como estrategia para desplazar el uso de químicos agrícolas, se aplica la bioinoculación de microorganismos promotores de crecimiento vegetal. En este contexto, el objetivo fue evaluar el efecto promotor del crecimiento vegetal de cinco cepas de microorganismos sobre seis especies de plantas. Se seleccionó un hongo (Trichoderma harzianum) y un grupo de bacterias (Enterobacter aerogenes, Azotobacter sp., Bacillus mycoides y Microbacterium sp.) para evaluar su efecto sobre la germinación, crecimiento y asimilación de plantas de yuca Manihot esculenta, arroz Oryza sativa, girasol Helianthus annuus L., arveja Pisum sativum L., lechuga Lactuca sativa L. y papaya Carica papaya L. Para el montaje del bioensayo se dispuso de 18 macetas para cada planta. Cada tratamiento se realizó por triplicado incluyendo el control. Se elaboró el inoculo sembrando una alícuota de cada microorganismos en $200 \mathrm{~mL}$ de caldo nutritivo, y de este se agregaron $20 \mathrm{~mL}$ a cada maceta por tratamiento. Se evaluaron las siguientes variables: número y longitud de raíces, hojas y tallo, área foliar y biomasa raíz y tallo. Los biopreparados a partir de Microbacterium sp. y $B$. mycoides, aceleraron la germinación de las plantas sin importar la especie. Los tratamientos de Azotobacter sp., T. harzianum y E. aerogenes no fueron efectivos para las plantas de yuca y arroz. La bioinoculación de Microbacterium sp., B. mycoides y E. aerogenes favoreció el crecimiento, desarrollo y rendimiento de las plantas de arroz y arveja.

Palabras clave: bioinoculante, inóculo, germinación, desarrollo, bioensayo. 


\begin{abstract}
The indiscriminate use of agrochemicals to increase crop productivity is not an environmentally friendly practice. As a strategy to replace the use of agricultural chemicals, we propose the use of bioinoculation of plant growth-promoting microorganisms. In this context, the objective of this research was to evaluate the effect of five plant growth-promoting microorganism strains on six plants. A fungus (Trichoderma harzianum) and a group of bacteria (Enterobacter aerogenes, Azotobacter sp., Bacillus mycoides and Microbacterium sp.) were selected to evaluate the effect of germination, growth and assimilation in cassava (Manihot esculenta), rice (Oryza sativa), sunflower (Helianthus annuus), peas (Pisum sativum L.), lettuce (Lactuca sativa L.), and papaya (Carica papaya L.). Bioassays included 18 pots per plant species, and each treatment was replicated three, including the control. The inoculum was prepared by seeding $200 \mathrm{~mL}$ of nutrient broth with an aliquot of each microorganism, then, adding $20 \mathrm{~mL}$ of inoculum to each pot per treatment. The following variables were evaluated: root number and length, leaves and stems, leaf area, and root and stem biomass. The bioinoculation of Microbacterium sp. and B. mycoides accelerated plant germination regardless of the species. The treatments with Azotobacter sp., T. harzianum and E. aerogenes were not effective in cassava and rice crops. The bioinoculation of Microbacterium sp., B. mycoides and E. aerogenes favored growth, development and yield in rice and pea crops.
\end{abstract}

Keywords: Bioinoculum, inoculum, germination, development, bioassay.

\section{INTRODUCCIÓN}

El suelo es uno de los hábitats microbianos que presenta mayor biodiversidad. Se estima que alberga cerca de $10^{9}$ y $10^{4}$ microorganismos y especies diferentes por gramo de suelo, respectivamente. Estos microorganismos pueden interactuar en la rizosfera con las raíces de las plantas, de modo que los exudados radicales, ricos en compuestos orgánicos les aportan gran variedad de nutrientes para llevar a cabo sus actividades metabólicas (Rodríguez, 2013).

La multifuncionalidad de los microorganismos en los sistemas agrícolas, se expresa de acuerdo con una serie de factores bióticos, como la competencia con otros microorganismos, la composición biológica del suelo, el reconocimiento plantamicroorganismo y viceversa. Igualmente, factores abióticos, como la climatología, las características físicas y químicas del suelo, que influyen directamente en el tipo de interacción de estos organismos y la expresión de los efectos benéficos o detrimentales, son determinantes en el desarrollo de las especies vegetales (Radjacommare et al., 2010).

La interacción de microorganismos rizosféricos, como los hongos formadores de micorrizas arbusculares (AMF), hongos del género Trichoderma Nonfried, 1894 y Pseudomonas Migula, 1894, usualmente catalogados como agentes de control biológico (BCA) y microorganismos promotores del crecimiento vegetal, dependen de este tipo de factores para expresar sus potenciales efectos benéficos; sin embargo, las interacciones entre los microorganismos son complejas y se pueden presentar efectos sinérgicos que potencialicen los beneficios para la planta ó por el contrario, efectos antagónicos o, simplemente, que no ocurra ningún efecto (Cano, 2011).

Los microorganismos promotores de crecimiento vegetal (PCV), son un grupo de diferentes especies que pueden incrementar el crecimiento y productividad en las plantas. Entre los organismos más 
conocidos, están las especies pertenecientes a los géneros Rhizobium sp., Pseudomonas sp. M, y Azospirillum. Los microorganismos PCV, pueden clasificarse en dos grupos: (i) microorganismos promotores de crecimiento en plantas, donde el mismo afecta a las plantas suprimiendo otros microorganismos. Los mecanismos que estos tienen que ver con su propio metabolismo (solubilizando fosfatos, produciendo hormonas o fijando nitrógeno), los cuales afectan directamente el metabolismo de la planta (incrementando la toma de agua y minerales), mejorando el desarrollo radicular, incrementan la actividad enzimática de la planta o propician que otros microorganismos benéficos actúen de mejor manera sobre las plantas (Bashan y Holguin, 1998). (ii) Bacterias promotoras de crecimiento en plantas con capacidad de control biológico, las cuales promueven el crecimiento de la planta al suprimir los fitopatógenos. El grupo de microorganismos conocido como PGPR (Plant Growth Promoting Rhizobacteria) fue definido por Kloepper et al. (1989) como microorganismos que colonizan la raíz y estimulan significativamente el crecimiento de plantas.

Pese a numerosos estudios con este grupo de microorganismos, aún no está totalmente establecido el mecanismo por el cual, los microorganismos PCV promueven el crecimiento de las plantas, pero se puede inferir que pueden inducir el crecimiento vegetal directa e indirectamente. La influencia directa incluye la producción de fitohormonas, por ejemplo, ácido indolacético (AIA) del grupo de las auxinas, ácido giberélico $\left(\mathrm{GA}_{3}\right)$, citoquininas y ácido abscísico (ABA) o bien, la capacidad de producir la enzima 1-aminociclopropano 1-carboxilato (ACC) desaminasa, que reduce el nivel de etileno en las raíces. Además, los mecanismos directos incluyen la liberación de fosfatos (Beltrán, 2014), micronutrientes y la fijación biológica de nitrógeno. Los efectos indirectos se deben a la modificación del ambiente rizosférico y su ecología, actuando como agentes de biocontrol de fitopatógenos mediante la liberación de sideróforos, $\beta$-1, 3-gluconasas, quitinasas, antibióticos, entre otras (Angulo et al., 2014).

Además de producir o cambiar la concentración de fitohormonas, algunas especies de este grupo de microorganismos participan en la fijación asimbiótica de Nitrógeno $\left(\mathrm{N}_{2}\right)$, pueden desarrollar antagonismo contra microorganismos fitopatógenos, pueden producir sideróforos, solubilizar el fosfato mineral y otros nutrientes. Según Kloepper et al. (1989) los microorganismos PCV pueden ser: bioprotectores (supresión de enfermedades de plantas), biofertilizantes (aumentar la capacidad de adquisición de nutrientes) y bioestimulantes (producción de fitohormonas).

Teniendo en cuenta las propiedades de los microorganismos PCV y la versatilidad ambiental que los caracteriza, se han realizado varios esfuerzos en todo el mundo para formular y utilizar estos microorganismos como biofertilizantes. Los biofertilizantes son productos que contienen microorganismos vivos o partes activa de ellos, los cuales ejercen uno o varios efectos benéficos en las plantas, usando diferentes mecanismos.

El uso de microorganismos PCV permite mejorar o reducir las diversas formas de fertilización química al suelo, e incluso en pesticidas químicos, generando de esta forma prácticas más amigables con la salud del suelo y el ambiente, mientras que al mismo tiempo se beneficia la planta y la economía del agricultor.

Por lo tanto, el objetivo de esta investigación fue analizar la capacidad PCV de cinco microorganismos sobre seis especies de plantas de fácil crecimiento y de elevados consumos de energía en la Región Caribe Colombiana, para luego evaluar la capacidad PCV de los microorganismos sobre las especies vegetales. 


\section{MATERIALES Y MÉTODOS}

Obtención del biopreparado. Se utilizaron cinco microorganismos, un hongo Trichoderma harzianum $(\mathrm{N})$ y cuatro bacterias (Enterobacter aerogenes Hormaeche, 1960; Azotobacter sp. Beijerinck, 1901; Bacillus mycoides Flügge, 1886 y Microbacterium sp. Orla-Jensen, 1919) para preparar el inóculo. Para confirmar la morfología de las bacterias, se realizó tinción Gram, y para el hongo se utilizó coloración con azul de lactofenol, para luego realizar análisis bajo el microscopio.

Confirmada la morfología microscópica y macroscópica, se tomó una alícuota de cada cepa y se inoculó en $200 \mathrm{~mL}$ de caldo nutritivo, se incubó a temperatura ambiente $\left(30^{\circ} \mathrm{C}\right)$ durante ocho días. Pasados los ocho días, se transfirieron $10 \mathrm{~mL}$ del cultivo en caldo nutritivo a 12 tubos de Eppendorf. El contenido de los tubos se centrifugó a 360rpm durante 10 minutos, se retiró el sobrenadante y se escogió el pellet (biomasa), con el propósito de disolverlo en $200 \mathrm{~mL}$ de solución salina para obtener el inoculante final para cada tratamiento.

Montaje del bioensayo. Se utilizaron 18 recipientes (macetas) amplios con $20 \mathrm{~cm}$ de profundidad y $30 \mathrm{~cm}$ de ancho por cada planta. Cada recipiente contenia $3000 \mathrm{~g}$ de suelo más o menos fértil proveniente del jardín principal de la Universidad Popular del Cesar (Sede Sabanas). El bioensayo se realizó con seis especies de plantas: yuca $(\mathrm{Y})$ Manihot esculenta, arroz (A) Oryza sativa, girasol (G) Helianthus annuus, arveja (Ar) Pisum sativum L., (L) lechuga Lactuca sativa y papaya (P) Carica papaya L. El proceso se realizó por triplicado para cada tratamiento en cada planta.

Las semillas de cada especie se sembraron a un centímetro de profundidad, teniendo presente que el número de semillas a utilizar varió de acuerdo con el tamaño de las mismas, de modo que para girasol, arveja, arroz y papaya se utilizaron 10 semillas por maceta, para lechuga 25 y para la yuca solo cuatro estacas. Sumado a esto, se utilizó un tratamiento control para poder comparar los resultados finales. Luego, a cada recipiente se le adicionaron $30 \mathrm{~mL}$ del biopreparado, atendiendo al tratamiento rotulado en el mismo.

Seguimiento del bioensayo. Al esquema de tratamiento completo se le brindó las condiciones mínimas para su germinación, evitando que los factores externos influyeran sobre los resultados. El seguimiento de las semillas germinadas fue diario por un periodo de 16 días, para arroz y girasol, mientras que para las plantas de yuca, papaya, arveja y lechuga, el periodo fue de 22 días. Deacuerdo con Polon (2007) se caracterizó el efecto de los tratamientos sobre las plantas en cuatro grupos: efecto positivo, el tratamiento favorece las variables medidas sobre el tratamiento control; efecto negativo, el tratamiento no favorece las variables y las cifras son inferiores al tratamiento control; ningún efecto, la medición de las variables es igual tanto en el tratamiento evaluado como en el tratamiento control; y por último, no hubo crecimiento, la germinación de las semillas es cero.

Variables evaluadas. Se evaluaron las siguientes variables: número de raíces (NR), número de hojas $(\mathrm{NH})$, longitud de las raíces (LR) y longitud del tallo (LT), área foliar (AF) y biomasa de la raíz (BR) y biomasa del tallo (BT). Para calcular la biomasa tanto del tallo como de la raíz, se envolvieron por separado en papel periódico y se llevó al horno a $65^{\circ} \mathrm{C}$ por un periodo de 24 horas.

El porcentaje de germinación (G) de la semilla se determinó teniendo en cuenta la ecuación propuesta por Araya et al. (2000), que establece la relación entre el número de semillas germinadas y el número de semillas sembradas, expresadas en porcentaje. 


$$
\mathrm{G}=\left(\frac{\text { Semillas germinadas }}{\text { Semillas sembradas }}\right) * 100
$$

Ya seca la planta, se separó la raíz del tallo y se pesaron en conjunto con las estructuras homólogas pertenecientes a las otras plantas del mismo tratamiento. Este procedimiento se realizó con cada tratamiento y su control respectivo, los resultados se dividieron por el número de semillas germinadas en cada maceta, obteniendo la biomasa del tallo y la raíz.

\section{RESULTADOS Y DISCUSIÓN}

Efecto de los microorganismos sobre la germinación de las semillas. De las cepas inoculadas, el
$50 \%$ incrementó la germinación de las semillas, el $20 \%$ no tuvo ningún efecto sobre las mismas y el $30 \%$ redujo la germinación (Tabla 1). En las plantas de Yuca, las especies de microorganismos que mostraron efectos benéficos en la brotación de los esquejes fueron Microbacterium sp. (0), Bacillus mycoides (F) y Azotobacter sp. (B) con porcentajes superiores al control del 25, 50 y 50\%, respectivamente. Lo anterior se debe a la relación estrecha entre la superficie radicular de la yuca y estos microorganismos rizosféricos; sin embargo, Trichoderma harzianum (N) y Enterobacter aerogenes $(\mathrm{H})$ redujeron la brotación de los esquejes de yuca en un 25\%, lo que permite inferir que estos microorganismos PCV producen fitohormonas específicas que retardan e inhiben la brotación de las semillas de yuca.

Tabla 1. Germinación de las semillas e incremento de la germinación (IG) en relación con el control. (Y= Yuca; A= Arroz; Ar= Arveja; G= Girasol; L= Lechuga; P= Papaya).

\begin{tabular}{|c|c|c|c|}
\hline Tratamiento & Planta & Germinación + (\%) & IG con relación al control (\%) \\
\hline \multirow{6}{*}{ Microbacterium sp. } & Y & 75 & 25 \\
\hline & A & 100 & 0 \\
\hline & $\mathrm{Ar}$ & 78 & 34 \\
\hline & $\mathrm{G}$ & 60 & 60 \\
\hline & $\mathrm{L}$ & 28 & -28 \\
\hline & $\mathrm{P}$ & 80 & 10 \\
\hline \multirow{6}{*}{ Bacillus mycoides } & Y & 100 & 50 \\
\hline & $\mathrm{A}$ & 100 & 0 \\
\hline & $\mathrm{Ar}$ & 89 & 45 \\
\hline & $\mathrm{G}$ & 100 & 100 \\
\hline & $\mathrm{L}$ & 56 & 0 \\
\hline & $\mathrm{P}$ & 100 & 30 \\
\hline \multirow{6}{*}{ Azotobacter sp. } & $\mathrm{Y}$ & 100 & 50 \\
\hline & $\mathrm{A}$ & 95 & -5 \\
\hline & $\mathrm{Ar}$ & 78 & 34 \\
\hline & $\mathrm{G}$ & 0 & 0 \\
\hline & $\mathrm{L}$ & 0 & -56 \\
\hline & $\mathrm{P}$ & 80 & 10 \\
\hline \multirow{6}{*}{ Trichoderma harzianum } & $\mathrm{Y}$ & 25 & -25 \\
\hline & A & 90 & -10 \\
\hline & $\mathrm{Ar}$ & 67 & 23 \\
\hline & $\mathrm{G}$ & 0 & 0 \\
\hline & $\mathrm{L}$ & 16 & -40 \\
\hline & $\mathrm{P}$ & 90 & 20 \\
\hline Enterobacter aerogenes & $\begin{array}{c}\mathrm{Y} \\
\mathrm{A} \\
\mathrm{Ar} \\
\mathrm{G} \\
\mathrm{L} \\
\mathrm{P}\end{array}$ & $\begin{array}{c}25 \\
95 \\
33 \\
30 \\
0 \\
70 \\
\end{array}$ & $\begin{array}{c}-25 \\
-5 \\
-11 \\
30 \\
-56 \\
0\end{array}$ \\
\hline
\end{tabular}


En las semillas de arroz, Azotobacter sp. (B), Trichoderma harzianum (N) y Enterobacter aerogenes $(\mathrm{H})$, tuvieron efectos negativos en la germinación, reduciéndola en 5, 10 y 5\% en su orden. Aunque el porcentaje de germinación reducido no es alto, se evidencia la afectación en el proceso, lo cual puede convertirse en pérdidas económicas en caso de utilizarse inóculos con base en estos microorganismos en cultivos arroceros. En otras investigaciones, Rojas y Moreno (2008) han encontrado que Azotobacter sp. (B) tiene efectos benéficos sobre la germinación y crecimiento del cultivo de arroz, debido a la estimulación integral de los componentes del crecimiento en estas plántulas a través de la producción de grandes cantidades de baba capsular, concluyendo que la aplicación de biopreparados en cultivos de arroz a partir de este microorganismo, puede aumentar la estabilidad del mismo.

Las semillas de arveja, a diferencia de las demás, presentan dos cotiledones, por ello, presentaron un crecimiento lento, sin embargo, hubieron microorganismos que aumentaron el porcentaje de germinación de ésta especie vegetal. Microbacterium sp. (0) y Azotobacter sp. (B) aumentaron un $34 \%$, Trichoderma harzianum (N) un $23 \%$ y Bacillus mycoides (F) 45\%, respecto al control, donde se evidenció el aumento en la germinación que indujeron estas especies PCV sobre las semillas de arveja. Al respecto, Chanway et al. (1989) evaluaron el efecto de la inoculación con nueve cepas de bacterias de los géneros Pseudomonas sp. (M) y Serratia sp. Bizio, 1823 en dos especies de leguminosas y encontraron efectos positivos significativos en la germinación de lenteja (Lens esculenta) causados por la inoculación de las cepas, con incrementos de hasta $38,9 \%$ con la mejor cepa en comparación con el testigo (sin inocular), efecto que se debe a la velocidad con la que estos microorganismo colonizan la raíz y multiplican la espermósfera como respuesta a los exudados radicales; no así en el cultivo de arveja (Pisum sativum L.), en el cual no detectaron efectos significativos, puesto que estos microorganismos presentan una alta tasa de especificidad o relación microorganismo-planta, por ello, los efectos del mismo microorganismo sobre las especies vegetales no siempre es la misma; datos similares a los encontrados en el presente experimento, y aunque sean microorganismos diferentes, comparten la particularidad de no ser simbiontes.

Las semillas de girasol también mostraron un proceso de germinación medianamente lento, pero al momento de la inoculación, respondieron positivamente al tratamiento con base en Bacillus mycoides (F), puesto que el aumento en el porcentaje de germinación con relación al control fue de 100\%, lo que permite inferir que el microambiente rizosférico era adecuado para este microorganismo. Dicho aumento se debe posiblemente a la producción de giberelinas, las cuales interrumpen la fase de latencia de las semillas y las hacen germinar (Pabón y Castaño, 2012). Trichoderma harzianum (N) y Azotobacter sp. (B) no produjeron ningún tipo de efecto sobre las semillas de girasol, puesto que la semilla para poder germinar requiere de una exposición a bajas temperaturas de $5^{\circ} \mathrm{C}$ durante $24 \mathrm{~h}$, lo anterior conllevó a que el porcentaje de germinación se mantuvo igual al del control. Por otra parte, los tratamiento de Microbacterium sp. (0) y Enterobacter aerogenes $(\mathrm{H})$, mostraron efectos levemente benéficos, pero en menor porcentaje que el tratamiento de Bacillus mycoides (F).

El efecto de los microorganismos en la germinación de la lechuga, fue negativo en comparación con el control sin inocular (Tabla 1), a excepción de Bacillus mycoides (F) que no tuvo efecto alguno sobre el incremento en la germinación, lo que indica que las especies PCV evaluadas no estimulan la germinación en estas semillas, puesto que la alta concentración de giberelinas retardó el crecimiento de las plantas de lechuga, inhibiendo la estimulación de la formación de raíces y hojas; por ende, estos microorganismos no resultarían ser efectivas en campo. La anterior razón, también puede deberse a la condición ambiental a la que se sometió el ensayo, pues las plantas de lechuga crecen óptimamente 
en condiciones ambientales entre 14 y $16^{\circ} \mathrm{C}$, condiciones que no fueron brindadas en esta investigación, siendo que el crecimiento de las plantas se dio en condiciones ambientales normales de la ciudad de Valledupar $\left(33\right.$ y $\left.35^{\circ} \mathrm{C}\right)$.

Por último, en las semillas de papaya, cuatro de los cinco microorganismos PCV evaluados mostraron influencia positiva sobre la germinación de las semillas, con valores de 10, 10, 20 y 30\% para Microbacterium sp. (0), Azotobacter sp. (B) Trichoderma harzianum (N) y Bacillus mycoides $(\mathrm{F})$, respectivamente, lo cual permite deducir que las semillas de papaya asimilan fácilmente los productos que sintetizan los microorganismos aislados, los cuales son utilizados por la semilla para acelerar el proceso de germinación. Un factor que influye positivamente en el aumento del porcentaje de germinación con relación al control, es la particularidad de las semillas de papaya al poseer una capa de mucigel o mucílago que rodea y protege la semilla, y que al tiempo favorece la adherencia de microorganismos y el contacto con sustancias orgánicas de fácil asimilación por parte de la misma como es el caso de las giberelinas u otras hormonas que faciliten la germinación de las semillas de papaya.

En la Tabla 1 se puede apreciar que así como existieron tratamientos que influyeron positivamente en el aumento de la germinación de las semillas, otros influyeron de forma negativa, reduciendo la velocidad y el porcentaje de germinación, incluso, hubieron tratamientos que no ejercieron ningún efecto sobre la germinación de las plantas.

Efecto de los tratamientos sobre la velocidad de germinación de las semillas. Así como los tratamientos tuvieron efectos benéficos sobre el porcentaje de germinación de las semillas, también lo expresaron acelerando la germinación en algunos casos, retardándolas en otros, o incluso no influyendo en el proceso.
Como se puede apreciar en la Tabla 2, el tratamiento Microbacterium sp. (0) generó efectos positivos en yuca, girasol y papaya. En arveja, este tratamiento expresó un efecto altamente benéfico, puesto que ésta semilla no germinó en el tratamiento sin inocular (control) y en las semillas de arroz, no hubo efecto alguno, puesto que el comportamiento fue el mismo que en el control. Lo anterior, se debe a la incapacidad de este microorganismo para controlar la concentración de ácido abscísico, resultados semejantes a los obtenidos por Kloepper et al. (1989), donde concluyen luego de varios análisis, que el ácido abscísico es un inhibidor de crecimiento y germinación natural presente en las plantas. En lechuga, este tratamiento retrasó la germinación de las semillas, pues en el tratamiento sin inocular (control), se evidenció el inicio de la germinación al quinto día de montado el bioensayo, y en el tratamiento M1 al séptimo día. Esto se debe a que no hubo liberación de fitohormonas al medio, por ende no se estimuló la germinación ni el desarrollo de las plántulas. Estos resultados concuerdan con los reportes sobre la producción de factores de crecimiento (auxinas, giberelinas y citoquininas) obtenidos por Capote et al. (2009).

Tabla 2. Velocidad en el inicio de la germinación de las semillas. (M1) Microbacterium sp., (M2) Bacillus mycoides, (M3) Azotobacter sp., (M4) Trichoderma harzianum, (M5) Enterobacter aerogenes. (Y= Yuca; $\mathrm{A}=$ Arroz; Ar= Arveja; G= Girasol; L= Lechuga; $\mathrm{P}=$ Papaya).

\begin{tabular}{lcccccc}
\hline & \multicolumn{9}{c}{ Germinación (días) } \\
\cline { 2 - 7 } Tratamientos realizados & Y & A & G & Ar & L & P \\
Control & 4 & 2 & 5 & 0 & 5 & 5 \\
Microbacterium sp. (M1) & 3 & 2 & 3 & 10 & 7 & 4 \\
Bacillus mycoides (M2) & 2 & 2 & 2 & 8 & 5 & 3 \\
Azotobacter sp (M3) & 3 & 3 & 3 & 0 & 0 & 4 \\
Trichoderma harzianum (M4) & 5 & 4 & 4 & 0 & 0 & 4 \\
Enterobacter aerogenes (M5) & 5 & 3 & 6 & 12 & 0 & 5 \\
\hline
\end{tabular}


El tratamiento B. mycoides (F) aumentó la velocidad de germinación en las semillas de yuca, girasol, arveja y papaya. En las semillas de arroz y lechuga no expresó ningún tipo de efecto (Tabla 2), lo que permite inferir que B. mycoides (F) es un microorganismo PCV con alto potencial, puesto que las semillas en la que éste microorganismo no influyó, tienen unos requerimientos especiales, como lo son, las bajas temperaturas para las plantas de lechuga y la alta concentración de agua en los cultivos de arroz, pese a estos requerimientos específicos, las diferencias en los días de germinación no fueron distantes (no excedieron los dos días).

De acuerdo con Lara et al. (2007), Azotobacter sp. es una especie de microorganismo PCV con capacidad de fijación biológica y asimbiótica del $\mathrm{N}_{2}$ atmosférico; a su vez, tienen habilidad para la producción de fitohormonas que favorecen la germinación, crecimiento, desarrollo y estabilidad de muchos cultivos de plantas (Vallejo et al., 2007). En yuca acelero la brotación y en arroz, girasol y papaya aceleró el inicio de la germinación con respecto al control. Pese a que éste microorganismo tiene la capacidad de fijar el $\mathrm{N}_{2}$ (Córdova et al., 2009), y que la arveja es una planta leguminosa, debería suponerse una influencia positiva sobre la germinación, pero se asume este efecto negativo a la presencia de dos cotiledones, los cuales influyen también en la germinación.

Cabe aclarar, que el tratamiento (M3) no influyó positivamente sobre la velocidad de germinación en las plantas de lechuga y arveja, pero tampoco se obtuvo una expresión negativa sobre estas especies vegetales. El efecto neutral de éste microorganismo, se debe a que no existe relación específica con los exudados radicales de éstas plantas, lo que conlleva a la incapacidad de estimular la germinación a través de fitohormonas.
El tratamiento Trichoderma harzianum (N) influyó positivamente sobre las semillas de girasol y papaya, reduciendo el tiempo de germinación en un día. En los ensayos de arveja y lechuga no influyó de ninguna forma, por ende, entre éste microorganismo y estas especies, la relación inicial es neutral. Sobre las semillas de arroz y yuca, (N) expresó una influencia negativa, aumentando los días para el inicio de la germinación de estas especies vegetales.

Por último, E. aerogenes (M5) permite obtener resultados bastante variados, en las especies vegetales de yuca, arroz y girasol. M5 influye negativamente, aumentando los días para iniciar la germinación. En el tratamiento control sin inocular, las semillas de arvejas no germinaron, sin embargo, bajo este tratamiento hubo emergencia de radicula al día 12. Para el caso de las semillas de lechuga, en el tratamiento control hubo germinación desde el quinto día, sin embargo, bajo el tratamiento M5 no hubo germinación alguna. En las semillas de papaya, este tratamiento es neutral, al no afectar ni promover la germinación ni la velocidad de inicio de la misma.

Relación planta y microorganismos. En yuca, Bacillus mycoides (F) y Microbacterium sp. (0) presentan un mecanismo de acción directa como la producción de fitohormonas (auxinas y citoquinas). En este caso, hubo clorosis en las hojas debido a la escases de fosfato del suelo.

En cuanto a las plantas de arroz, todas las especies de microorganismos utilizados tuvieron efectos benéficos, en especial, en la reestimulación del número de raíces. Cabe mencionar, que Microbacterium sp. y Bacillus mycoides promueven el número de raíces a través de la producción de citoquinas, así mismo Microbacterium sp. fue mayor en la elongación del tallo (producción de 
auxinas), lo que significa que Bacillus mycoides ejerce un efecto productor de auxinas.

Por el contrario, la planta de arveja es lenta en el proceso de germinación, lo cual se comprobó por qué no hubo semillas germinadas en el tratamiento control sin inoculación. Sin embargo, se evidenció que Bacillus mycoides (F), Microbacterium sp. (0) y Enterobacter aerogenes $(\mathrm{H})$, presentaron un efecto promotor por la producción de giberelinas y además, estimula la producción de auxinas a nivel de elongación de tallo y raíces, también favorecen la producción de citoquinas aumentando el número de raíces y hojas. No obstante, la más efectiva fue la cepa de E. aerogenes $(\mathrm{H})$.

En las plantas de girasol, B. mycoides (F) fue el más efectivo en la producción de auxinas y citoquinas, evidenciándose dicha afirmación en el número de raíces. Sin embargo, Microbacterium sp. (0) y Azotobacter sp. (B) también presentaron un efecto benéfico por la producción de estas mismas fitohormonas notables, en aumento de longitud de tallo, en el número de hojas y longitud de raíces.

En lechuga, el tratamiento con T. harzianum (N) demostró un efecto benéfico tanto en la producción de auxinas como en la producción de citoquinas, demostrando en estas plantas, un notable incremento en el número de raíces y en la longitud del tallo y raíces; sin embargo, B. mycoides (F) tuvo el mismo efecto pero menos apreciado. Cabe notar que con el tratamiento de E. aerogenes $(H)$ se afectó significativamente la germinación de la semilla, disminuyendo el porcentaje en más del $50 \%$ (Tabla 1).

En las plantas de papaya hubo efecto benéfico directo en el aumento del número de hojas y raíces (producción de citoquinas y auxinas) apreciables con mayor facilidad en los tratamientos Microbacterium sp. (0), Azotobacter sp. (B) y Bacillus mycoides (F).

\section{Efecto de los microorganismos PCV en el} crecimiento y desarrollo de las plantas. No todas las cepas de microorganismos evaluados, tuvieron efectos positivos sobre las plantas, y no todas las plantas respondieron de la misma forma a los tratamientos, es decir, mientras que el tratamiento beneficiaba una variable o aspecto, podría tener influencia negativa sobre otro en la misma planta, toda la afirmación anterior se debe a las comparaciones que se hicieron con el control o tratamiento sin inocular.

Como se aprecia en las Tablas 3 y Tabla 4, en las plantas de yuca, el efecto de Microbacterium sp. (0) y B. mycoides (F) favoreció el crecimiento de las raíces en cuanto al largo frente a las plántulas del control. El tratamiento a partir de Azotobacter sp. (B) produjo efectos negativos, efecto que se debe a la alta capacidad de producción de giberelinas por parte de este microorganismo, dicha capacidad fue reportada en Cuba por Hernández et al. (1992), donde mencionan la presencia de giberelinas en altas concentraciones en medios de cultivos de Azotobacter sp. (B). 
Tabla 3. Efecto de los tratamientos en las plantas: positivo (+), negativo (-), Ningún efecto ( $=$ ) y No hubo crecimiento $(0)$.

\begin{tabular}{|c|c|c|c|c|c|c|c|c|}
\hline \multirow{2}{*}{ Planta } & \multirow{2}{*}{ Tratamiento } & \multicolumn{7}{|c|}{ Efectos } \\
\hline & & LR & NR & BR & $\mathrm{NH}$ & $\mathrm{AF}$ & $\mathrm{BF}$ & LT \\
\hline \multirow{6}{*}{ Yuca } & CONTROL & 1,47 & 4,00 & 10,53 & 5,00 & 0,20 & 0,53 & 4,47 \\
\hline & M1 & + & - & 0 & + & $=$ & - & - \\
\hline & M2 & + & $=$ & - & - & + & - & - \\
\hline & M3 & - & - & 0 & - & + & - & - \\
\hline & M4 & $=$ & - & - & + & - & - & - \\
\hline & M5 & 0 & 0 & 0 & 0 & 0 & 0 & 0 \\
\hline \multirow{6}{*}{ Arroz } & CONTROL & 8,13 & 6,00 & 13,77 & 2,00 & 0,00 & 9,77 & 16,90 \\
\hline & M1 & + & + & + & + & + & + & + \\
\hline & M2 & + & + & + & + & + & + & + \\
\hline & M3 & 0 & 0 & 0 & 0 & 0 & 0 & 0 \\
\hline & M4 & 0 & 0 & 0 & 0 & 0 & 0 & 0 \\
\hline & M5 & + & + & + & + & + & + & + \\
\hline \multirow{6}{*}{ Girasol } & CONTROL & 4,13 & 26,00 & 10,00 & 5,00 & 0,30 & 37,32 & 9,53 \\
\hline & M1 & + & + & - & + & - & - & - \\
\hline & M2 & + & + & - & + & $=$ & - & + \\
\hline & M3 & - & + & 0 & + & + & - & - \\
\hline & M4 & - & - & - & + & - & - & - \\
\hline & M5 & + & + & - & + & $=$ & - & - \\
\hline \multirow{6}{*}{ Lechuga } & CONTROL & 1,30 & 1,00 & 0,43 & 3,00 & 0,17 & 1,50 & 1,50 \\
\hline & M1 & + & $=$ & - & + & - & - & $=$ \\
\hline & M2 & + & + & 0 & $=$ & $=$ & - & - \\
\hline & M3 & 0 & 0 & 0 & 0 & 0 & 0 & 0 \\
\hline & M4 & + & + & 0 & $=$ & + & - & + \\
\hline & M5 & 0 & 0 & 0 & 0 & 0 & 0 & 0 \\
\hline \multirow{6}{*}{ Papaya } & CONTROL & 5,80 & 6,00 & 2,73 & 4,00 & 0,30 & 13,53 & 5,60 \\
\hline & M1 & + & - & - & + & $=$ & - & - \\
\hline & M2 & - & $=$ & 0 & + & + & - & - \\
\hline & M3 & - & + & - & + & + & - & - \\
\hline & M4 & - & - & - & $=$ & - & - & $=$ \\
\hline & M5 & - & - & - & $=$ & - & - & - \\
\hline \multirow{6}{*}{ Arveja } & CONTROL & 0,00 & 0,00 & 0,00 & 0,00 & 0,00 & 0,00 & 0,00 \\
\hline & M1 & + & + & + & + & + & + & + \\
\hline & M2 & + & + & + & + & + & + & + \\
\hline & M3 & 0 & 0 & 0 & 0 & 0 & 0 & 0 \\
\hline & M4 & 0 & 0 & 0 & 0 & 0 & 0 & 0 \\
\hline & M5 & + & + & + & + & + & + & + \\
\hline
\end{tabular}


Tabla 4. Variación promedio del largo de raíz (LR), número de raíz (NR), biomasa raíz (BR), número de hojas (NH), área foliar (AF), biomasa foliar (BF) y largo tallo (LT). \pm 1 a desviación estándar.

\begin{tabular}{|c|c|c|c|c|c|c|c|c|}
\hline Tratamientos & Planta & $\begin{array}{c}\mathbf{L R} \\
(\mathbf{c m})\end{array}$ & NR & $\begin{array}{c}\text { BR } \\
(\mathrm{mg})\end{array}$ & NH & $\begin{array}{c}\mathrm{AF}\left(\mathrm{cm}^{2} /\right. \\
\mathrm{mg})\end{array}$ & $\begin{array}{c}\text { BF } \\
(\mathrm{mg})\end{array}$ & $\begin{array}{c}\text { LT } \\
(\mathbf{c m})\end{array}$ \\
\hline \multirow{6}{*}{ CONTROL } & $\mathrm{Y}$ & $1,47 \pm 0,25$ & $4,00 \pm 1,00$ & $10,53 \pm 1,22$ & $5,00 \pm 1,00$ & $0,20 \pm 0,10$ & $0,53 \pm 0,12$ & $4,47 \pm 0,59$ \\
\hline & A & $8,13 \pm 2,15$ & $6,00 \pm 1,00$ & $13,77 \pm 0,15$ & $2,00 \pm 1,73$ & $0,00 \pm 0,00$ & $9,77 \pm 1,99$ & $16,90 \pm 3,53$ \\
\hline & $\mathrm{Ar}$ & $0,00 \pm 0,00$ & $0,00 \pm 0,00$ & $0,00 \pm 0,00$ & $0,00 \pm 0,00$ & $0,00 \pm 0,00$ & $0,00 \pm 0,00$ & $0,00 \pm 0,00$ \\
\hline & G & $4,13 \pm 0,67$ & $26,00 \pm 7,21$ & $10,00 \pm 2,00$ & $5,00 \pm 1,73$ & $0,30 \pm 0,05$ & $51,00 \pm 9,54$ & $9,53 \pm 1,44$ \\
\hline & $\mathrm{L}$ & $1,30 \pm 0,66$ & $1,00 \pm 1,00$ & $0,43 \pm 0,15$ & $3,00 \pm 1,00$ & $0,17 \pm 0,06$ & $1,50 \pm 0,17$ & $1,50 \pm 0,20$ \\
\hline & $\mathrm{P}$ & $5,80 \pm 1,40$ & $6,00 \pm 1,00$ & $2,73 \pm 0,32$ & $4,00 \pm 0,00$ & $0,30 \pm 0,10$ & $13,53 \pm 1,16$ & $5,60 \pm 1,13$ \\
\hline \multirow{6}{*}{$\begin{array}{c}\text { Microbacterium sp. } \\
\text { (M1) }\end{array}$} & $\mathrm{Y}$ & $2,00 \pm 0,70$ & $1,00 \pm 0,00$ & $0,00 \pm 0,00$ & $26,00 \pm 1,00$ & $0,20 \pm 0,10$ & $0,22 \pm 0,00$ & $2,50 \pm 0,20$ \\
\hline & A & $12,00 \pm 5,29$ & $5,00 \pm 1,00$ & $0,02 \pm 0,00$ & $2,33 \pm 1,53$ & $0,00 \pm 0,00$ & $0,01 \pm 0,00$ & $25,50 \pm 1,65$ \\
\hline & Ar & $6,00 \pm 0,00$ & $20,33 \pm 0,00$ & $0,49 \pm 0,09$ & $6,00 \pm 1,00$ & $0,17 \pm 0,12$ & $0,23 \pm 0,02$ & $5,33 \pm 2,08$ \\
\hline & G & $7,47 \pm 1,92$ & $28,33 \pm 3,21$ & $0,01 \pm 0,01$ & $6,00 \pm 1,73$ & $0,20 \pm 0,10$ & $0,08 \pm 0,01$ & $8,00 \pm 0,82$ \\
\hline & $\mathrm{L}$ & $2,00 \pm 0,36$ & $1,00 \pm 0,00$ & $0,01 \pm 0,01$ & $4,00 \pm 2,65$ & $0,15 \pm 0,10$ & $0,01 \pm 0,00$ & $1,50 \pm 0,48$ \\
\hline & $\mathrm{P}$ & $5,97 \pm 0,61$ & $1,00 \pm 0,00$ & $0,05 \pm 0,02$ & $4,67 \pm 1,53$ & $0,30 \pm 0,02$ & $0,11 \pm 0,03$ & $5,00 \pm 2,00$ \\
\hline \multirow{6}{*}{$\begin{array}{l}\text { Bacillus mycoides } \\
\text { (M2) }\end{array}$} & Y & $2,70 \pm 0,46$ & $4,00 \pm 1,00$ & $0,01 \pm 0,00$ & $4,00 \pm 1,00$ & $0,23 \pm 0,06$ & $0,08 \pm 0,01$ & $2,00 \pm 1,00$ \\
\hline & A & $9,50 \pm 0,10$ & $9,00 \pm 1,00$ & $0,02 \pm 0,01$ & $3,00 \pm 0,00$ & $0,33 \pm 0,06$ & $0,01 \pm 0,00$ & $4,80 \pm 0,10$ \\
\hline & $\mathrm{Ar}$ & $10,47 \pm 0,15$ & $13,00 \pm 4,58$ & $0,05 \pm 0,00$ & $9,00 \pm 1,00$ & $0,30 \pm 0,10$ & $0,03 \pm 0,01$ & $13,00 \pm 1,73$ \\
\hline & G & $5,00 \pm 0,20$ & $32,33 \pm 6,43$ & $0,01 \pm 0,00$ & $6,00 \pm 1,00$ & $0,30 \pm 0,20$ & $0,05 \pm 0,02$ & $11,17 \pm 0,90$ \\
\hline & $\mathrm{L}$ & $1,44 \pm 0,14$ & $2,00 \pm 1,00$ & $0,00 \pm 0,01$ & $3,00 \pm 1,00$ & $0,17 \pm 0,08$ & $0,01 \pm 0,00$ & $1,46 \pm 0,01$ \\
\hline & $\mathrm{P}$ & $4,67 \pm 0,45$ & $6,00 \pm 1,00$ & $0,00 \pm 0,00$ & $5,00 \pm 0,00$ & $0,31 \pm 0,02$ & $0,01 \pm 0,00$ & $4,70 \pm 0,26$ \\
\hline \multirow{6}{*}{$\begin{array}{c}\text { Azotobacter sp. } \\
\text { (M3) }\end{array}$} & $\mathrm{Y}$ & $1,23 \pm 0,12$ & $2,00 \pm 0,00$ & $0,00 \pm 0,00$ & $3,00 \pm 0,00$ & $0,22 \pm 0,10$ & $0,02 \pm 0,01$ & $2,90 \pm 0,00$ \\
\hline & A & $6,40 \pm 1,21$ & $12,00 \pm 1,00$ & $0,01 \pm 0,00$ & $2,00 \pm 1,00$ & $0,27 \pm 0,06$ & $0,01 \pm 0,01$ & $16,90 \pm 0,20$ \\
\hline & $\mathrm{Ar}$ & $0,00 \pm 0,00$ & $0,00 \pm 0,00$ & $0,00 \pm 0,00$ & $0,00 \pm 0,00$ & $0,00 \pm 0,00$ & $0,00 \pm 0,00$ & $0,00 \pm 0,00$ \\
\hline & G & $3,70 \pm 0,20$ & $26,33 \pm 4,51$ & $0,00 \pm 0,00$ & $6,00 \pm 1,00$ & $0,33 \pm 0,07$ & $0,03 \pm 0,01$ & $10,90 \pm 0,10$ \\
\hline & $\mathrm{L}$ & $0,00 \pm 0,00$ & $0,00 \pm 0,00$ & $0,00 \pm 0,00$ & $0,00 \pm 0,00$ & $0,00 \pm 0,00$ & $0,00 \pm 0,00$ & $0,00 \pm 0,00$ \\
\hline & $\mathrm{P}$ & $4,93 \pm 0,29$ & $12,67 \pm 2,08$ & $0,02 \pm 0,02$ & $5,00 \pm 1,73$ & $0,32 \pm 0,02$ & $0,01 \pm 0,00$ & $5,60 \pm 0,44$ \\
\hline \multirow{6}{*}{$\begin{array}{c}\text { Trichoderma } \\
\text { harzianum (M4) }\end{array}$} & Y & $1,47 \pm 0,21$ & $1,00 \pm 0,00$ & $0,01 \pm 0,01$ & $12,00 \pm 2,65$ & $0,11 \pm 0,02$ & $0,02 \pm 0,01$ & $3,00 \pm 0,62$ \\
\hline & A & $5,80 \pm 0,40$ & $8,00 \pm 3,00$ & $0,01 \pm 0,01$ & $2,00 \pm 0,00$ & $0,00 \pm 0,00$ & $0,01 \pm 0,01$ & $15,30 \pm 0,10$ \\
\hline & Ar & $0,00 \pm 0,00$ & $0,00 \pm 0,00$ & $0,00 \pm 0,00$ & $0,00 \pm 0,00$ & $0,00 \pm 0,00$ & $0,00 \pm 0,00$ & $0,00 \pm 0,00$ \\
\hline & G & $2,13 \pm 0,25$ & $17,00 \pm 2,00$ & $0,02 \pm 0,01$ & $6,00 \pm 1,73$ & $0,01 \pm 0,00$ & $0,02 \pm 0,00$ & $6,37 \pm 0,42$ \\
\hline & $\mathrm{L}$ & $2,00 \pm 0,26$ & $3,00 \pm 2,00$ & $0,00 \pm 0,00$ & $3,00 \pm 0,00$ & $0,27 \pm 0,12$ & $0,01 \pm 0,01$ & $1,80 \pm 0,10$ \\
\hline & $\mathrm{P}$ & $2,50 \pm 0,20$ & $5,00 \pm 2,00$ & $0,01 \pm 0,01$ & $4,00 \pm 1,00$ & $0,23 \pm 0,02$ & $0,06 \pm 0,02$ & $5,60 \pm 0,36$ \\
\hline \multirow{6}{*}{$\begin{array}{c}\text { Enterobacter aerogenes } \\
\text { (M5) }\end{array}$} & $\mathrm{Y}$ & $0,00 \pm 0,00$ & $0,00 \pm 0,00$ & $0,00 \pm 0,00$ & $0,00 \pm 0,00$ & $0,00 \pm 0,00$ & $0,00 \pm 0,00$ & $0,00 \pm 0,00$ \\
\hline & A & $4,83 \pm 0,12$ & $9,00 \pm 1,00$ & $0,01 \pm 0,00$ & $2,00 \pm 0,00$ & $0,00 \pm 0,00$ & $0,66 \pm 0,56$ & $8,33 \pm 0,12$ \\
\hline & $\mathrm{Ar}$ & $9,93 \pm 0,15$ & $24,00 \pm 1,00$ & $0,02 \pm 0,01$ & $5,00 \pm 0,00$ & $0,22 \pm 0,02$ & $0,01 \pm 0,02$ & $5,57 \pm 0,32$ \\
\hline & G & $4,80 \pm 0,26$ & $27,00 \pm 2,00$ & $0,01 \pm 0,00$ & $7,00 \pm 1,00$ & $0,30 \pm 0,06$ & $0,02 \pm 0,01$ & $7,30 \pm 0,20$ \\
\hline & $\mathrm{L}$ & $0,00 \pm 0,00$ & $0,00 \pm 0,00$ & $0,00 \pm 0,00$ & $0,00 \pm 0,00$ & $0,00 \pm 0,00$ & $0,00 \pm 0,00$ & $0,00 \pm 0,00$ \\
\hline & $\mathrm{P}$ & $4,70 \pm 0,10$ & $4,00 \pm 1,00$ & $1,33 \pm 0,31$ & $4,00 \pm 0,00$ & $0,21 \pm 0,10$ & $0,01 \pm 0,01$ & $4,47 \pm 0,15$ \\
\hline
\end{tabular}


Hernandez et al. (1992) mencionan la capacidad retardadora del crecimiento de las giberelinas cuando su concentración es superior a otras fitohormonas. El efecto negativo se refleja en la disminución de la longitud de las raices, mientras que tratamientos como Trichoderma harzianum $(\mathrm{N})$ y E. aerogenes $(\mathrm{H})$ no produjeron efecto alguno y no estimularon el crecimiento, respectivamente.

El tratamiento a partir de E. aerogenes $(\mathrm{H})$ afecto significativamente el crecimiento y desarrollo de las plantas yuca, puesto que bajo este microorganismo no se produjo crecimiento durante los 22 días que duró el bioensayo. Todos los tratamientos evaluados afectan el crecimiento del tallo de las plantas yuca y la biomasa foliar.

En las plántulas de arroz, Microbacterium sp. (0), B. mycoides (F) y E. aerogenes $(\mathrm{H})$ influyeron positivamente en el crecimiento y desarrollo de las mismas. Los resultados fueron positivos en todas las variables (Tabla 3). Cabe resaltar de este primer grupo de microorganismos PCV que Microbacterium sp. (0) tiene la capacidad de solubilizar el fosfato y hacerlo más disponible para las plantas (Beltrán, 2014), por ello, favoreció el crecimiento, desarrollo y estabilidad de las plantas de arroz. Por otra parte, los tratamientos de $T$. harzianum (N) y Azotobacter sp. (B) inhibieron el crecimiento de las mismas, sin embargo, Sakthiselvan et al. (2014) han sugerido que T. harzianum (N) puede favorecer el crecimiento vegetal, y cuando se mezcla con carbón de bajo rango (CBR) generan un efecto positivo sobre algunas propiedades químicas del suelo aumentando la solubilización de contaminantes y la capacidad de absorción de los mismos.

Los tratamientos que se evaluaron en esta investigación, produjeron efectos benéficos en el número de hojas para las plantas de girasol. T. harzianum (N) produjo efectos negativos en todas las variables medidas, a excepción de la antes mencionadas. A las plántulas de girasol evaluadas bajo el tratamiento de Azotobacter sp. (B) se les dificultó medir la biomasa de la raíz por lo pequeñas que eran, además, el dato que arrojó la balanza fue muy pequeño y lejano del control.

Las plantas de lechuga, pese a que se encontraban bajo condiciones ambientales no óptimas, se evidenció un crecimiento y desarrollo positivo en algunos tratamientos con respecto al control no inoculado, gracias a la inducción recibida por alguno de los tratamientos; sin embargo, casos como los de Azotobacter sp. (B) y E. aerogenes $(\mathrm{H})$ inhibieron totalmente el crecimiento de esta planta, impidiendo al mismo tiempo la germinación de las semillas durante los 22 días que duró el ensayo biológico. Puede apreciarse en la Tabla 3, que el tratamiento a partir de T. harzianum $(\mathrm{N})$ fue el que obtuvo mejores resultados; por esta razón, la bioinoculación de este tratamiento podría aumentar la productividad en las plantas de lechuga bajo condiciones óptimas para esta planta, aunque haya resultado negativo en la biomasa foliar.

Aunque todos los tratamientos aceleraron la germinación de las semillas de papaya, no resulta efectivo el uso de tratamientos a partir de estos microorganismos, puesto que frente al control, no hubo mejorías significativas en cuanto a las demás variables como largo de tallo, número de hojas, raíces, entre otras. Resulta valido afirmar que los tratamientos no afectaron la cantidad de hojas en estas plantas, a pesar de que T. harzianum (N) y E. aerogenes $(\mathrm{H})$ no influyeron sobre la cantidad de hojas, Microbacterium sp. (0), B. mycoides (F) y Azotobacter sp. (B) indujeron aumentos en la cantidad de hojas de las plantas de papaya con respecto al control sin inocular.

En el caso de las plantas de arveja, no hubo crecimiento en el tratamiento sin inocular (control), los tratamientos T. harzianum (N) y Azotobacter sp. (B) no produjeron efecto alguno, dado que bajo estos tratamientos tampoco hubo crecimiento, no obstante, se observó crecimiento de las plántulas de arveja en los tratamientos 
a partir de Microbacterium sp. (0), B. mycoides (F) y Azotobacter sp. (B). El no crecimiento de la leguminosa bajo el tratamiento de Azotobacter sp. (B) que tiene capacidad de fijación asimbiótica y biológica del Nitrógeno (Obando et al., 2013) se debe a que ésta bacteria es muy utilizada como un complemento biofertilizante, para reducir las cantidades de fertilizantes nitrogenados de aplicación tradicional, más no como única fuente de nitrógeno, que a diferencia de las bacterias simbióticas como el caso de Rhizobium leguminosarum (F) fijan menos cantidades de nitrógeno y expresan una relación más estrecha con las plantas leguminosas.

Variables evaluadas. Con esta investigación se demostró que existen microorganismos con capacidad promotora de crecimiento vegetal, pero que no expresan dicha capacidad en todas las especies de plantas, destacándose por los mejores resultados los tratamientos a partir de Microbacterium sp. (0), B. mycoides (F) y E. aerogenes $(\mathrm{H})$ para las plantas de arroz y arveja. Hubieron también microorganismos PCV que inhibieron el crecimiento y desarrollo de las plántulas, como es el caso de E. aerogenes $(\mathrm{H})$ en yuca; T. harzianum (N) y Azotobacter sp. (B) en arroz y arveja y por último Azotobacter sp. (B) y E. aerogenes $(\mathrm{H})$ en las plantas de lechuga.

En la Tabla 5 se presenta el Análisis de Varianza (ANDEVA) para las variables de las plantas evaluadas. Se observó que existen diferencias significativas entre las plantas evaluadas con respecto al largo de raíz (LR), número de raíz (NR) y biomasa raíz (BR) y la biomasa foliar (BF) con un $\mathrm{p} \leq 0,05$.

Adicionalmente, no existen diferencias significativas en el número de hojas $(\mathrm{NH})$ para las plantas arroz, girasol y Papaya, el área foliar en el girasol y la papaya al igual que el largo del tallo en esta última.

El análisis de correlación de Pearson presentado en la Tabla 6. indica que existe una relación altamente significativa entre el número de raices (NR) y la longitud de las raices (LR) al igual que la relación entre el largo del tallo (LT) con el número de raices (NR) y el largo de las raices (LR) y similar a la relación entre área foliar $(\mathrm{AF})$ y el número de raices (NR).

Tabla 5. ANDEVA de las variables largo de raíz (LR), número de raíz (NR), biomasa raíz (BR), número de hojas (NH), área foliar (AF), biomasa foliar (BF) y largo tallo (LT).

\begin{tabular}{|c|c|c|c|c|c|c|c|c|}
\hline \multirow{2}{*}{ PLANTA } & \multirow{2}{*}{ ANOVA } & \multicolumn{7}{|c|}{ Análisis estadístico de las variables evaluadas } \\
\hline & & LR & NR & BR & $\mathrm{NH}$ & $\mathrm{AF}$ & $\mathrm{BF}$ & LT \\
\hline \multirow{2}{*}{$\mathrm{Y}$} & $\mathrm{F}$ & 17,557 & 12,600 & 223,306 & 160,738 & 4,214 & 51,514 & 21,911 \\
\hline & $\mathrm{P}$ & 0,000 & 0,000 & 0,000 & 0,000 & 0,019 & 0,000 & 0,000 \\
\hline \multirow{2}{*}{ A } & $\mathrm{F}$ & 3,722 & 7,929 & 25008,063 & 0,463 & 60,900 & 65,380 & 62,580 \\
\hline & $\mathrm{P}$ & 0,029 & 0,002 & 0,000 & 0,797 & 0,000 & 0,000 & 0,000 \\
\hline \multirow{2}{*}{$\mathrm{Ar}$} & $\mathrm{F}$ & 441,184 & 100,011 & 83,737 & 135,600 & 12,767 & 202,845 & 64,626 \\
\hline & $P$ & 0,000 & 0,000 & 0,000 & 0,000 & 0,000 & 0,000 & 0,000 \\
\hline \multirow{2}{*}{ G } & $\mathrm{F}$ & 12,879 & 3,476 & 74,847 & ,601 & 1,034 & 4,782 & 18,386 \\
\hline & $\mathrm{P}$ & 0,000 & 0,036 & 0,000 & 0,701 & 0,441 &, 012 & 0,000 \\
\hline \multirow{2}{*}{ L } & $\mathrm{F}$ & 23,134 & 4,100 & 24,038 & 5,919 & 5,958 & 230,915 & 42,871 \\
\hline & $\mathrm{P}$ & 0,000 & 0,021 & 0,000 & 0,006 & 0,005 & 0,000 & 0,000 \\
\hline \multirow{2}{*}{ P } & $\mathrm{F}$ & 10,379 & 23,582 & 114,973 & 0,619 & 1,781 & 405,322 & 0,819 \\
\hline & $\mathrm{P}$ & 0,000 & 0,000 & 0,000 & 0,688 & 0,191 & 0,000 & 0,559 \\
\hline
\end{tabular}


Tabla 6. Correlación de Pearson entre largo de raíz (LR), número de raíz (NR), biomasa raíz (BR), número de hojas $(\mathrm{NH})$, área foliar $(\mathrm{AF})$, biomasa foliar (BF) y largo tallo (LT).

\begin{tabular}{c|cccccc}
\hline VARIABLES & NR & BR & NH & AF & LT & BF \\
\hline LR & $0,461^{* *}$ & 0,092 & 0,091 & $-0,002$ & $0,071^{* *}$ & 0,069 \\
NR & & $-0,007$ & 0,139 & $0,249^{*}$ & $0,415^{* *}$ & 0,066 \\
BR & & & 0,005 & $-0,025$ & $-0,035$ & 0,015 \\
NH & & & & 0,065 & 0,01 & 0,156 \\
AF & & & & & 0,096 & $-0,018$ \\
LT & & & & & & 0,044 \\
\hline
\end{tabular}

* Significativa $(\mathrm{p} \leq 0,05)$ (bilateral). ${ }^{* *}$ Altamente significativa $(\mathrm{p} \leq 0,01)$

Para las plantas de girasol y papaya, no resulta efectivo el uso de tratamientos a partir de estos microorganismos, a pesar que produjeron efectos positivos en algunas de las variables evaluadas, no resulta positivo el uso de las mismas, por lo cual se recomienda el análisis de los antagonismos entre los microorganismos, para poder evaluar mezclas de microorganismos en posibles investigaciones, y así poder determinar la forma en que un microorganismo favorece o inhibe el efecto de otro sobre las plantas.

\section{CONCLUSIONES}

Los microorganismos Microbacterium sp. (0) y $B$. mycoides (F) indujeron y aceleraron la germinación de las semillas sin importar la especie. Los microorganismos Azotobacter sp. (B), T. harzianum $(\mathrm{N})$ y E. aerogenes $(\mathrm{H})$ no fueron efectivos para las plantas de yuca y arroz. Los microorganismos Microbacterium sp. (0), B. mycoides (F) y E. aerogenes $(\mathrm{H})$ favorecieron el crecimiento, desarrollo y rendimiento de las plantas de arroz y arveja; resultando estos últimos una excelente alternativa para favorecer el crecimiento de estas especies vegetales en prácticas amigables con el medio ambiente.

\section{REFERENCIAS BIBLIOGRÁFICAS}

ANGULO, V.; SANFUENTES, E.; RODRÍGUEZ, F.; SOSSA, K. 2014. Caracterización de rizobacterias promotoras de crecimiento en plántulas de Eucalyptus nitens. Rev. Arg. de Microbiología. 46(4): 338-347.

ARAYA, E.; GÓMEZ, L.; HIDALGO, N.; VALVERDE, R. 2000. Efecto de la luz y del ácido gilberalico sobre la germinación in vitro de (Alunus Acuminata). Agron. CR. 24(1):75 - 80.

BASHAN, Y.; G. HOLGUIN. 1998. A proposal for the division of "plant growth-promoting rhizobacteria" into two classifications: biocontrol-plant growth-promoting bacteria and plant growth-promoting bacteria. Soil Biol. Biochem. 30: 1225-1228.

BELTRÁN, M. 2014. La solubilización de fosfatos como estrategia microbiana para promover el crecimiento vegetal. CORPOICA Cienc. Tecnol. Agropecu. 101 - 113p.

CANO, M. 2011. Interacción de microorganismos benéficos en plantas: Micorrizas, Trichoderma spp. y Pseudomonas spp. una revisión. Revista U.D.C.A Actualidad \& Divulgación Científica. 14(2): 15-31.

CAPOTE, I.; ESCALONA, M.; DAQUINTA, M.; PINA, D.; GONZÁLES, J.; ARAGÓN, C. 2009. Efecto del análogo de brasinoesteroide (MH5) en la aclimatización de los brotes de Vriesea, propagadas en sistemas de inmersión temporal. Ciencia y Tecnología. 2(1):21 - 25.

CHANWAY, C.; RADLEY, R.; HOLL, F. 1989. Bacterial Inoculation of Lodgepole Pine, White Spruce, and Douglas -fir Grown in Containers. Department of Agriculture, Forest Service, Rocky Mountain Forest and Range Experiment Station. 93-97.

CÓRDOVA, Y.; RIVERA, M.; FERRERA, R.; OBRADOR, J.; CÓRDOVA, V. 2009. Detección de bacterias benéficas en el suelo con banano (Musa AAA simmondsk) cultivar "Gran enano" y si potencial para integrar un biofertilizante. Universidad y Ciencia. 25(3): 253 - 265.

HERNÁNDEZ, T.; DÍAZ, F.; GONZÁLEZ, J. 1992. Respuesta de las mejores variedades de arroz de ciclo medio al biofertilizante Azospirillum brasilense. Programa 
y Resúmenes VIII Seminario Científico. I Taller Internacional sobre Biofertilización en los Trópicos. INCA. La Habana, Cuba. 43p.

KLOEPPER, J.; LIFSHITZ, R.; ZABLOTOWITZ, R. 1989. Free living bacteria inocula for enhancing crop productivity. Trends in Biotechnology. 7: 39-43.

LARA, C.; VILLALBA, M.; OVIEDO, L. 2007. Bacterias fijadoras asimbióticas de nitrógeno de la zona agrícola de San Carlos. Córdoba, Colombia. Rev. Colomb. Biotecnol. 9(2): 6-14.

OBANDO, M.; RIVERA, D.; BONILLA, R. 2013. Respuesta fisiológica a la fertilización por Azotobacter chroococcum AC1 y fertilicación nitrogenada de síntesis sobre el Maíz (Zea mays) en el invernadero. Revista de la Sociedad Mexicana de Biotecnología y Bioingeniería. 17(1): 11-22.

PABÓN, J.; CASTAÑO, J. 2012. Identificación de hongos y bacterias en granos de arveja (Pisium sativum L.). agron. 20(1): 26-37.

POLON, R. 2007. Estudio de diferentes manejos de agua en el cultivo del arroz (Oryza sativa L.) y su influencia sobre la germinación, la masa seca, la altura de la planta y el rendimiento agrícola. Rev. Cul. Trop. 28(2): 101103.

RADJACOMMARE, C.; VENKATESAN, S.; SAMYAPPAN, R. 2010. Biological control of phytopathogenic fungi of vanilla through lytic action of Trichoderma sp and Pseudomonas fluorescens. Phytopathology and Plant Protection. 43(1): 1-17.

RODRÍGUEZ, C. 2013. Evaluación de microorganismos promotores de crecimiento vegetal en tomate (Solanum lycopersicum) variedad santa clara, aislados de residuos lignocelulósicos de higuerilla (Ricinus communis). Rev. Tec. Agro. 24(II): 23-31.

ROJAS, J.; MORENO, N. 2008. Producción y formulación de prototipos de un biofertilizante a partir de bacterias nativas asociadas al cultivo de arroz (Oryza sativa). Rev. colomb. Biotecnol. 10(2): 50-62.
SAKTHISELVAN, P.; NAVEENA, B. PARTHA, N. 2014. Molecular characterization of a Xylanaseproducing fungus isolated from fouled soil. Braz. J. Microbiol. 45(4): 1293-1302.

VALLEJO, M.; BONILLA, C.; CASTILLA, L. 2007. Evaluación de la asociación bacterias fijadoras de nitrógeno - líneas interespecíficas de arroz-nitrógeno, en Typic haplustalf. Ibagué, Colombia. Acta Agronómica. 57(1): 43-49. 